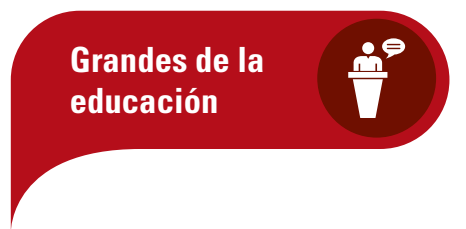

\title{
Don Bosco (1815-1888)
}

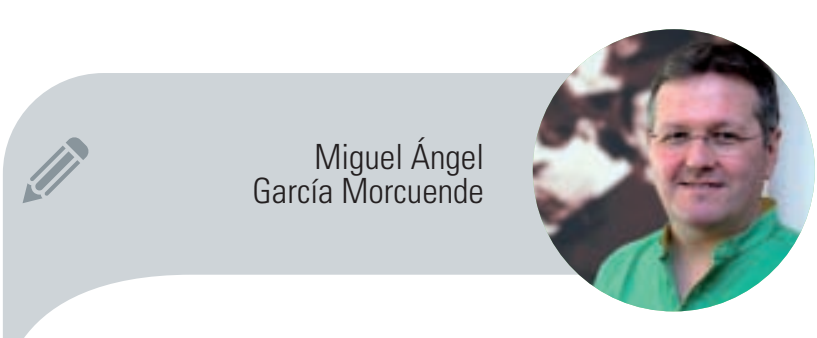

Responsable Oficina Escuela-Formación Profesional Salesianos Dirección General magarcia@sdb.org

El agitado siglo XIX italiano es el contexto de la fundación de la obra salesiana por parte de San Juan Bosco, familiarmente llamado Don Bosco. El carisma salesiano, su método educativo y su espiritualidad se traducen en una persistente preocupación por los adolescentes y jóvenes más vulnerables. Su multiforme experiencia de obras e iniciativas encuentra hoy gran actualidad psicológica y educativa, muchas de sus intuiciones siguen manteniendo una vigencia extraordinaria, incluso cuando se tiene en cuenta la época en la que fueron propuestas a los jóvenes y educadores de su tiempo.

El suyo era un proyecto casi utópico de elevación intelectual, profesional, física, moral y religiosa de los jóvenes de las clases populares y en riesgo, en particular: pobres, abandonados, en peligro o peligrosos, dependientes, aprendices, sin instrucción ni trabajo, expuestos a la calle o a la delincuencia. La vida y obra de este santo turinés cuyo bicentario del nacimiento se celebra este año 2015 es un album de escenas de esa compleja obra de educación, reeducación, recuperación e integración de los jóvenes marginados: "en lo que se refiere al bien de la juventud en peligro... yo me lanzo hasta la temeridad", decía.

\section{El deseo de prevenir mediante la educación}

La figura de Don Bosco educador está históricamente asociada a su Sistema Preventivo, un estilo de vida, una manera de ser y de actuar en medio de los niños, adolescentes y jóvenes: viene articulado como una experiencia espiritual, una propuesta de evangelización juvenil y una metodología pedagógica.

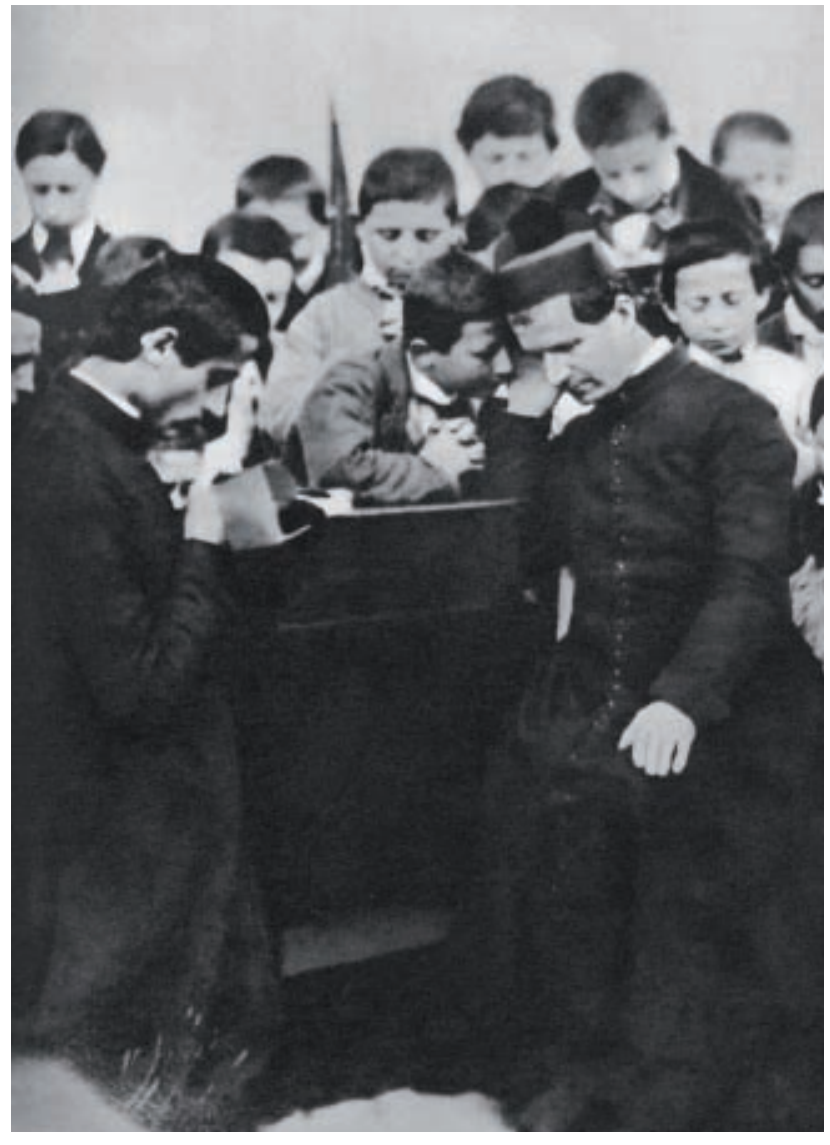

Su rica experiencia de educador y pedagogo se intuye en su praxis educativa personal y en la de los educadores por él formados. Su identidad como educador puede ser correctamente captada sólo en relación con su rica personalidad como sacerdote y fundador, legislador y organizador, educador y escritor popular.

La preventividad como criterio educativo significa aquella particular sensibilidad necesaria en el educador, expresada en una atención cuidada a todo lo que pueda constituir una experiencia irreversiblemente negativa para el joven, y como la confiada y constante promoción de experiencias que activan su proceso de maduración. En el primer caso, prevenir es evitar; en el segundo es anticipar. En ambas acentuaciones, lo preventivo es sólo una dimensión significativa de una propuesta más amplia, propuesta educativa, que no agota su riqueza y su complejidad.

La originalidad de Don Bosco está en acentuar, mendiante la educación, el crecimiento personal en su totalidad, y haber relacionado las opciones de fe a 


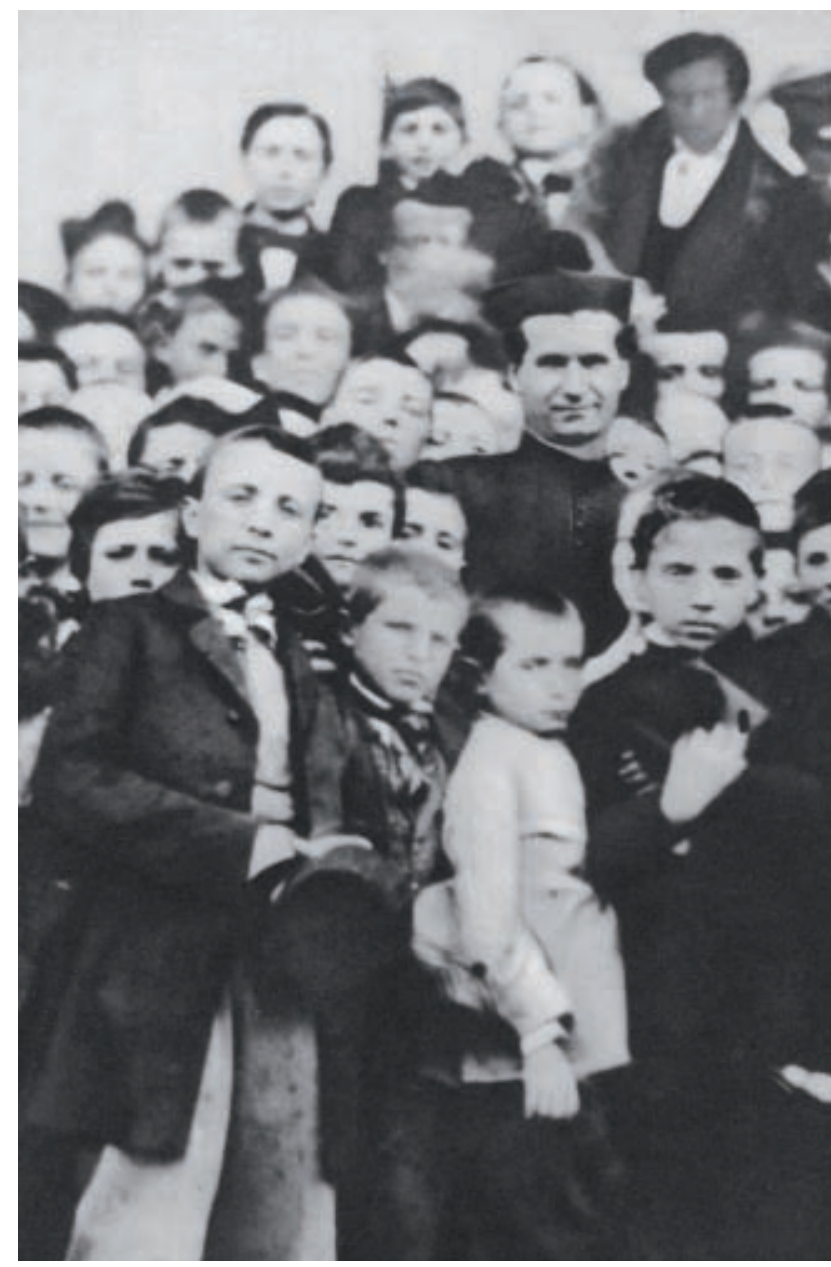

las que llegue el joven en este proceso, con sus compromisos civiles, a los que lo lleva su misma conciencia cristiana. Se trata, entonces, de un humanismo que está llamado a comprender y a realizar la plenitud de su vocación humana y su ser hijo de Dios: "Buenos cristianos y honestos ciudadanos".

\section{Las grandes coordenadas educativas}

Don Bosco humaniza su obra en base a tres dimensiones, un trinomio: «religión, razón y amabilidad». La pregunta religiosa constituye un elemento integrador y realizable, un aspecto unificador de todo su sistema de educación, y al mismo tiempo, una tarea a acompañar para toda una vida cristiana. Se sentía amigo entre los chicos y sacerdote siempre. Don Bosco está convencido de que no es posible una verdadera educación sin una apertura a lo transcendente. En segundo lugar, la razón, una persuasión inteligentemente motivada, que implica una comprensión objetiva del muchacho en sus circunstancias y la búsqueda de una relación adecuada y constructiva con él. Por último, el término cariño (amorevolezza), omnipresente en la literatura salesiana y principio supremo del Sistema Preventivo: podemos decir que es su contenido por excelencia y su síntesis metodológica. Es amor educativo, afecto dado y recibido, verdadera disponibilidad y presencia entre los jóvenes. Este amor abre el corazón y la inteligencia del joven al educador, hace amables sus propuestas e intervenciones y estimula su protagonismo y sus respuestas, su iniciativa y su creatividad.

\section{Un ecosistema rico en relaciones y familiaridad}

Razones psicológicas, históricas y religiosas llevaron a Don Bosco a la conclusión de que la educación es obra del ambiente y de la ejemplaridad de las relaciones personales, y que la mejor condición para ello es el cuidado de una estructura educativa esencialmente familiar. Familia, ambiente de familia, espontaneidad: son palabras muy cercanas en el diccionario salesiano. No hay educación sin afecto. No hay afecto, si no se crea un clima educativo.

Al utilizar el término "clima" se recurre a una metáfora tomada de la meteorología. Así como los climas influyen en el comportamiento y hasta en las características de los seres vivos, la atmósfera de una casa salesiana es una condición que explica el comportamiento, la identidad, los valores y las interacciones que desarrollan las personas. Esta convicción nace en el terreno práctico: Don Bosco lo sabía bien, especialmente cuando visita las cárceles, y recorre las calles y las plazas de Turín.

Un ambiente educativo está fuertemente influenciado por la calidad de las relaciones cotidianas entre las personas. Conservar esa "temperatura" en las relaciones es esencial: cuando éstas son recíprocas, en confianza cordial y afectuosa, el ambiente entero se beneficia. El tono informal de las relaciones, la convivencia (el recreo todos juntos) y la presencia física afectuosa permiten la confianza recíproca entre adulto y joven. Es muy saludable percibir la utilidad del esfuerzo de todos los que forman parte de ese ambiente, sentir una adecuada motivación para construir juntos, crear un auténtico sentido de pertenencia.

Un elemento también importante en un ambiente estimulante y formativo es la aceptación mutua de las personas, el respecto y el reconocimiento de lo que cada uno aporta. No sin olvidar el inconfundible papel que tiene el juego de estos binomios: el acercamiento y la acogida, el respeto y la sinceridad, la confianza y la paciencia, la disculpa y el perdón.

Por último, hay un proyecto de hombre encerrado en todo proyecto educativo, y este proyecto vale 


\section{Carta sobre los castigos (1883)}

La importancia de este escrito de Don Bosco no está tanto en la presentación de una casuística extensa sobre los castigos, como en los motivos y en las sugerencias más generales que la inspiran. El espíritu, la actitud educativa general, la valoración positiva de los jóvenes, el optimismo y la evidente predilección por una política pedagógica del amor. Palabras en armonía con todo su método.

«[...] En general, el sistema que nosotros tenemos que aplicar es el llamado preventivo que consiste en disponer los ánimos de nuestros alumnos de modo que, sin ninguna violencia exterior, lleguen a plegarse a hacer nuestros deseos. Con este sistema entiendo deciros que no hay que usar nunca medios coercitivos, sino siempre y sólo los de la persuasión y de la caridad. [...] Si sois verdaderos padres de vuestros alumnos, hace falta que también tengáis su corazón. Y no recurráis nunca a la represión o castigo sin razón y sin justicia; y sólo al modo de quien la usa a la fuerza y para cumplir un deber. [...]

1. No castiguéis nunca hasta después de haber agotado todos los otros medios. ¡Cuántas veces, queridos hijos míos, he debido persuadirme de esta gran verdad en mi larga carrera! Es más fácil, qué duda cabe, irritarse que conservar la calma; amenazar a un muchacho que persuadirlo; diría que resulta más cómodo para nuestra impaciencia y nuestra soberbia castigar a los que se nos resisten que corregirlos soportándolos con firmeza y benignidad. La caridad que os recomiendo es la que usaba S. Pablo hacia los fieles recién convertidos a la religión del Señor, y que con frecuencia le hacía llorar y suplicar cuando los veía menos dóciles y sin eco a su celo. Por eso yo recomiendo a todos los Directores que antes de nada tienen que usar la corrección paterna hacía nuestros queridos hijitos, y que ésta se haga en privado, o como se suele decir, en camera charitatis. Nunca se reprenda directamente en público a no ser que sea para impedir el escándalo o para repararlo si se hubiese dado. [...]

2. Procurar escoger en las correcciones el momento favorable. Cada cosa a su tiempo, dice el Espíritu Santo; y yo os digo que si hace falta una de esas dolorosas necesidades, se requiere también una gran prudencia para saber escoger el momento en el que esa represión sea beneficiosa. Porque las enfermedades del alma hay que tratarlas al menos como las del cuerpo. No hay nada tan peligroso como un remedio mal aplicado o fuera de tiempo. Un médico sabio aguarde que el enfermo esté en condiciones de soportarlo y para ello espera el momento favorable. Y nosotros lo podremos conocer sólo a partir de la experiencia perfeccionada por la bondad del corazón. Antes de nada, esperad a ser dueños de vosotros mismos; no dejéis traslucir que actuáis por malhumor o por violencia; porque entonces perderíais la autoridad, y el castigo se haría pernicioso. [...]

3. Eliminad cualquier idea que pueda hacer creer que se actúa por pasión. Cuando se castiga, difícilmente se conserva la calma que es necesaria para alejar cualquier duda de que se actúa para hacer sentir la propia autoridad y desahogar la propia pasión. Y con cuanto más despecho se actúa tanto más lo advierten los demás. El corazón de padre que nosotros debemos tener condena este modo de actuar. [...] Nuestro querido y manso S. Francisco, vosotros lo sabéis, se había impuesto a sí mismo una norma severa por la que su lengua no hablaría cuando el corazón estuviese agitado. Solía decir, efectivamente: "Temo perder en un cuarto de hora la poca dulzura que he procurado acumular en veinte años gota a gota, como el rocío, en el vaso de mi pobre corazón. Una abeja emplea muchos meses en hacer un poco de miel, que un hombre come de un bocado; y además, ¿para qué sirve hablar al que no escucha?". [...] Cuando hayáis logrado ganaros ese ánimo inflexible, os ruego que no sólo le dejéis la esperanza de vuestro perdón, sino también la de que pueda, con una buena conducta, borrar la mancha hecha por él con sus faltas.

4. Conducios de modo que dejéis al culpable la esperanza de que puede ser perdonado. Se obtendrá más con una mirada de amor, con una palabra de ánimo, que dé confianza a su corazón, que con muchos reproches que no hacen más que inquietar y reprimir su vigor. Yo he visto verdaderas conversiones con este sistema, que por otro camino parecían absolutamente imposibles. Sé que algunos de mis más queridos hijos no tienen vergüenza en confesar que fueron ganados así a nuestra Congregación y, por tanto, a Dios. Todos los jóvenes tienen sus días malos, iy vosotros también los tuvisteis! Y jay de nosotros! si no nos esforzamos por ayudarles a que los pasen aprisa y sin reproches. Algunas veces sólo hacer creer que no se piensa que lo ha hecho con malicia, basta para impedir que recaiga en la misma falta. Serán culpables, pero desean que no se les crea así. jFelices nosotros si sabemos servirnos de este medio para educar estos pobres corazones!

5. Qué castigos deben aplicarse y por quién. Recordemos que la fuerza castiga el vicio, pero no cura al vicioso. No se cultiva la planta doblándola con áspera violencia y por eso no se educa la voluntad gravándola con excesivo yugo. He aquí una serie de castigos que son los únicos que querría se usasen entre nosotros. Uno de los medios más eficaces de represión moral, es la mirada descontenta, severa y triste del superior, que hace ver al culpable, por poco corazón que tenga, que se encuentra en desgracia, y que le puede provocar al arrepentimiento y la enmienda. Corrección privada y paternal. No demasiados reproches; y hacerle sentir el disgusto de los padres y la esperanza de las recompensas. A la larga se sentirá obligado a mostrar gratitud y hasta generosidad.

[...] Esforcémonos en hacernos amar. 

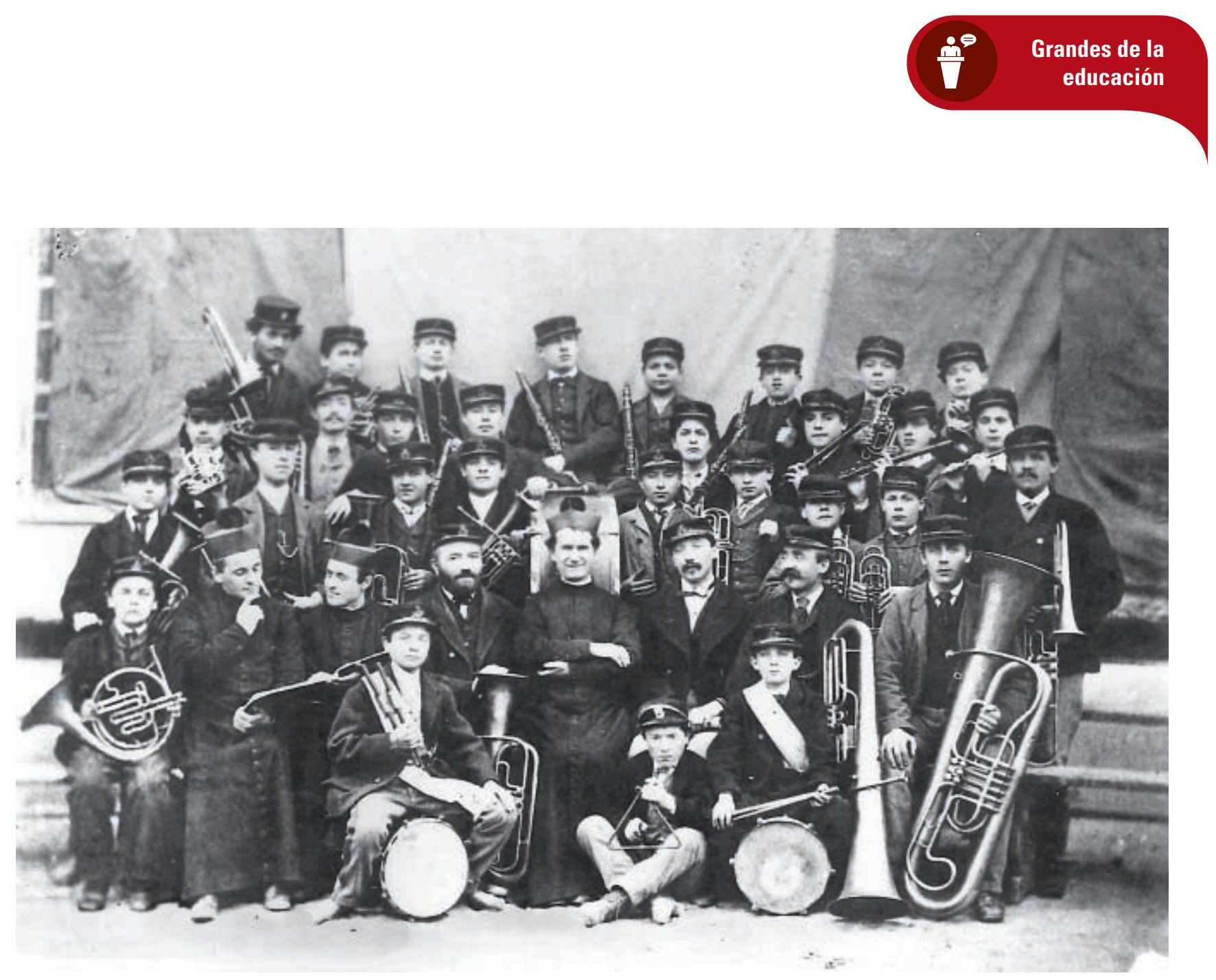

según construya o destruya al educando. A la base de la actitud y comportamiento pedagógicos y pastorales de Don Bosco, hay en él un concepto optimista de la persona. El realismo de aquella sociedad lacerada que vió y palpó, no lo alejó de la confianza en las posibilidades de recuperación de sus "pobres muchachos". Ésta, radicaba en una visión antropológica positiva, una fe en las reservas y posibilidades humanas y espirituales del sujeto educativo. Así es como, según Don Bosco, el educando siempre puede reencontrar en sí recursos personales que puestos en juego, juntamente con la "gracia", Io lleven a superar las limitaciones y condicionamientos deshumanizantes y a proponerse nuevas metas de superación y de conquista.

\section{Una criteriología pedagógica: la alegría}

Don Bosco intuyó la urgencia de crear en sus casas salesianas no sólo unas relaciones interpersonales con claras resonancias familiares, sino también espacios físicos distendidos y cordiales donde la nota dominante sea la libertad en la expresión y en la participación. En este sentido, una de las expresiones más ricas de la pedagogía de Don Bosco es la alegría; concedía espacio y dignidad al momento del recreo, la fiesta, el deporte, la música, el teatro y el patio. Todos ellos, lugares de la espontaneidad y de las relaciones donde el educador encuentra modos de intervención, tan sencillos en las expresiones como eficaces en los resultados para crear un clima de amistad y alegría.

La alegría es un antídoto perfecto contra la tristeza en todas sus formas. No se trata tanto de una conquista (nada se ve tan falso como el comportamiento de aquéllos que son alegres por obligación) como de un fruto: la alegría es la expresión de la felicidad, del estar en armonía consigo mismo, algo que sólo puede derivarse de estar en armonía con Dios y con los demás. Para los cristianos de todos los tiempos, los creyentes de toda edad, raza y condición han encontrado esa serenidad de corazón en el encuentro con Dios y la han expresado al Creador con su alegría vital. Don Bosco transmite a sus jóvenes y educadores que creemos en un Dios que no puede contenerse de júbilo por la felicidad de sus hijos, y que no desea ni expresa otra cosa; sino que también nosotros desbordemos alegría. Esta es la razón de la famosa expresión de Domingo Savio: "Nosotros demostramos la santidad, estando siempre alegres". Por eso invitaba a sus muchachos a explorar nuevos caminos, a valorar como positivo los eventos cotidianos, empapados de confianza en el Padre y en la entrega a los demás. La alegría salesiana es el amor disfrutado; es su primer fruto. Cuanto más grande es el amor, mayor es la alegría. 

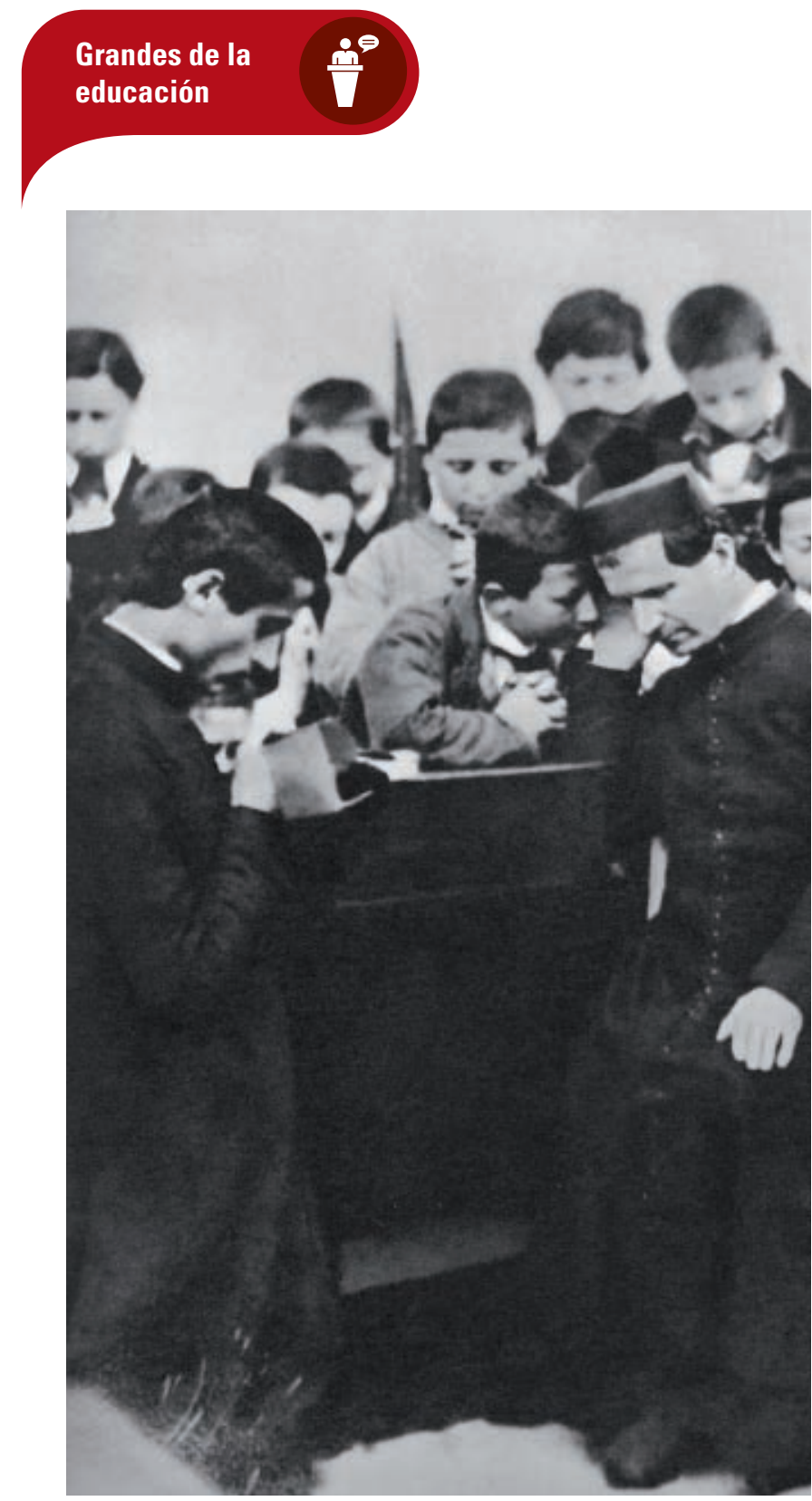

\section{Jóvenes nuevos, ¿educadores de siempre?}

Podríamos enumerar un gran número de cualidades consideradas esenciales en el educador de Don Bosco: amor pedagógico, tacto educativo, autoridad que se gana con la presencia activa y animadora, optimismo, equilibrio, paciencia y tantas otras.

Un educador ¿nace o se hace? Repensar de modo preventivo el perfil del educador, según Don Bosco, sólo cabe hacerlo desde alguien que hace de la educación una razón y una opción de vida. Las consideraciones anteriores no son aspectos instrumentales que atañen a la acción educativa; no exigen sólo ni principalmente un cambio de metodología, sino una nueva perspectiva, un nuevo tipo de formador. Hacer una relectura, en clave educativa salesiana, de la actual condición juvenil, su contexto y sus cambios acelerados requiere un perfil de educador vocacionado. De aquí arrancan las exigencias de competencia y excelencia de todo educador. Educar no puede reducirse a una profesión o un rol social sin un vivo interés personal. Un educador se hace asumiento la audacia de estar en el hábitat de las nuevas generaciones, en la onda de sus aspiraciones y problemas, para buscar con ellos los modos de traducir sus ideas en términos operativos, para estimular y alargar su capacidad de visión y de juicio crítico. Es, si se quiere, una vida inspirada en una concepción educativa.

El recuerdo de la fisonomía y actuación de Don Bosco suscitaron satisfacción. Se cumple en este caso que en toda persona adulta habitan sus educadores; tanto los que fallaron como los que nos has estimado con la «amorevolezza salesiana». La figura educativa del «santo de los jóvenes» nos recuerda que no es posible suscitar un afecto profundo leyendo un tratado sobre el arte de amar; el educador se hace encarnándose en una experiencia de amistad lograda y bien revestida de respeto.

El educador de Don Bosco no es sólo en una persona de referencia, sino es un facilitador del crecimiento de los individuos y del grupo entero. La pedagogía de Don Bosco ha retomado la sabia consideración de que quien educa, no puede esquivar la compañía educativa, no puede estar ausente de los círculos donde se toman, o se condicionan, las opiniones y las decisiones, no siempre comprensibles a los ojos de los adultos. La relación con los adolescentes está sometida a altibajos, infinidad de idas y venidas debido a su inestabilidad emocional, propia de su etapa evolutiva, de los condicionamientos sociofamiliares. Hemos de ser profundos conocedores de esto para no perder el pulso a la relación, ni valorar negativamente la respuesta del adolescente. Hay que permanecer a pesar de las turbulencias.

Sin darse tregua, Don Bosco halló modo de aunar energías, estrechar amistades, encontrar nuevos colaboradores, procurar sustentadores para sus obras en todos los estratos sociales. Con tal fin, asumimos también hoy la misión de ayudar a los jóvenes a jugar bien sus cartas y a utilizarlas para que puedan ganar la partida de la vida •

\section{(DA) DRRA SABER MÂS}

Bosco, J. (2015). Estad siempre alegres. Introducción, selección de textos y comentarios Miguel Ángel García Morcuende. Madrid: Narcea.

Braido, P. (2001). Prevenir, no reprimir. El sistema educativo de Don Bosco. Madrid: CCS.

Lenti, A. (2010-2012). Don Bosco: historia y carisma, vol. 3, Madrid: CCS.

Expósıto, A. (2015). Don Bosco hoy. Madrid: Editorial Romana. 